\title{
INFLUENCE OF TEMPERATURE AND ANNEALING ON GMR IN SPUTTERED PERMALLOY/Cu MULTILAYERS
}

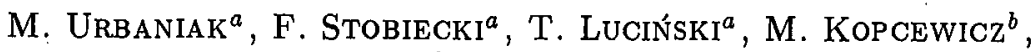 \\ A. GRABIAS ${ }^{b}$ AND J. AleKsIEJEW ${ }^{a}$ \\ ${ }^{a}$ Institute of Molecular Physics, Polish Academy of Sciences \\ Smoluchowskiego 17, 60-179 Poznań, Poland \\ ${ }^{b}$ Institute of Electronic Materials Technology \\ Wólczyńska 133, 01-919 Warszawa, Poland
}

The influence of temperature and annealing on giant magnetoresistance of $\mathrm{Si}(100) / \mathrm{Cu}(20 \mathrm{~nm}) / \mathrm{Py}(2 \mathrm{~nm}) /(\mathrm{Cu}(2 \mathrm{~nm}) / \mathrm{Py}(2 \mathrm{~nm}))_{100}$ multilayer $(\mathrm{Py}=$ $\left.\mathrm{Ni}_{83} \mathrm{Fe}_{17}\right)$ sputtered at room temperature in double face-to-face configuration is reported. It was found that giant magnetoresistance value, $\Delta R_{\mathrm{GMR}} / R_{\text {sat }}$ (where $R_{\text {sat }}$ is the resistance in saturation), monotonically decreases with increasing temperature $(4.5 \%$ at $173 \mathrm{~K}$ to about $1 \%$ at $373 \mathrm{~K})$. This results from the decrease in magnetic change of resistance, $\Delta R_{\mathrm{GMR}}$, and to the lesser extent from an increase in $R_{\text {sat }}$, though both of them are caused by the shortening of electrons mean free path. The observed almost linear decrease in giant magnetoresistance saturation field with increasing temperature is explained by temperature changes of magnetization profile. Vibrating sample magnetometer measurements revealed that the increase in temperature results in pronounced decrease in remnant to saturation magnetization ratio $\left(M_{\mathrm{r}} / M_{\mathrm{s}}\right)$ suggesting that at low temperatures magnetic bridges between Py layers play an important role in magnetization process. It is shown that proper annealing, by an annihilation of bridges and/or lateral decoupling, leads to an increase in giant magnetoresistance ratio from $3.4 \%$ in as deposited state to $4.7 \%$.

PACS numbers: $75.70 . \mathrm{Ak}, 75.70 . \mathrm{Pa}$

The discovery of giant magnetoresistance (GMR) in multilayered structures consisting of ferromagnetic layers separated by non-magnetic, conducting spacer has resulted in recent decade in materials that show considerable change of electrical resistance upon the application of weak magnetic field [1]. It is now relatively easy to produce systems exhibiting GMR field sensitivity exceeding $0.5 \% / \mathrm{Oe}[2,3]$. The emphasis has shifted therefore to technological aspects, like for example thermal stability of GMR systems which is necessary if they are to survive today's processing techniques required in microelectronics [4]. It turns 
out that $\mathrm{Py}\left(=\mathrm{Ni}_{83} \mathrm{Fe}_{17}\right) / \mathrm{Cu}$ multilayers are very suitable for obtaining high field sensitivities [5]. The reason is that a weak magnetic anisotropy of Py is accompanied by a relatively weak interlayer exchange coupling [1]. In this paper we present the analysis of the influence of temperature and annealing on the GMR effect in $\mathrm{Si}(100) / \mathrm{Cu}(20 \mathrm{~nm}) / \mathrm{Py}(2 \mathrm{~nm}) /(\mathrm{Cu}(2 \mathrm{~nm}) / \mathrm{Py}(2 \mathrm{~nm}))_{100}$ multilayer obtained by double face-to-face sputtering [6]. GMR ratio, defined as GMR $=100 \% \times\left(R_{\max }-R_{\min }\right) / R_{\min }$ [1] was measured with $\mathrm{DC}$ four-point method. The magnetization reversal processes were examined with vibrating sample magnetometer (VSM). The changes in the interface structure were investigated by conversion electron Mössbauer spectroscopy (CEMS) measurements performed at room temperature. $\mathrm{He}-6 \% \mathrm{CH}_{4}$ gas flow electron counter was used.

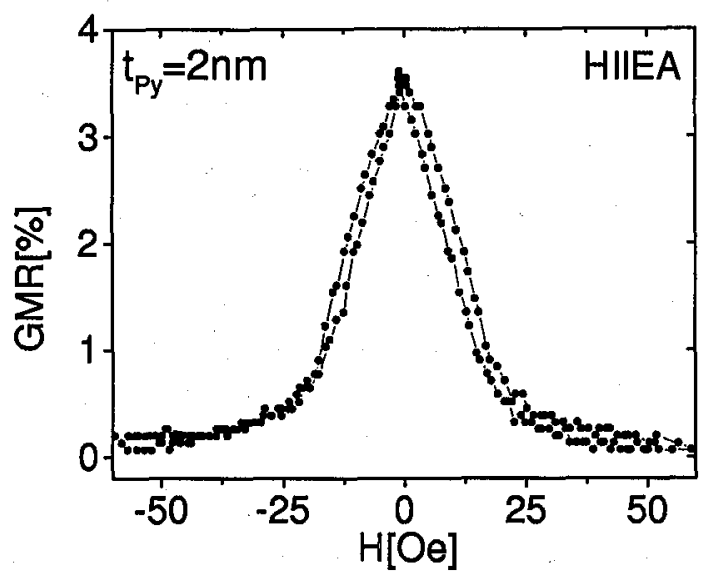

Fig. 1. Exemplary room temperature $\operatorname{GMR}(H)$ dependence obtained for the as-deposited sample with magnetic field and sensing current parallel to the easy axis direction.

An example of the GMR $(H)$ dependence is shown in Fig. 1. Figure 2 collects the GMR data obtained from temperature measurements of the as-deposited sample. It can be seen that although there is a considerable increase in resistance in saturation $\left(R_{\text {sat }}=R_{\text {min }}\right)$ with increasing temperature it is the decrease in magnetic change of resistance $\Delta R_{\mathrm{GMR}}$, i.e. $R_{\max }-R_{\min }$, which is the main cause of GMR ratio decrease. Physically, both of the observed dependencies come from the temperature driven decrease in electron mean free path on which GMR ratio directly depends [7]. It was shown previously that the GMR ratio in our samples scales proportionally with a fraction of antiferromagnetically (AF) coupled regions $F_{\mathrm{AF}}=\left(1-M_{\mathrm{r}} / M_{\mathrm{s}}\right)\left(M_{\mathrm{r}}\right.$ and $M_{\mathrm{s}}$ are remanence and saturation magnetization) [5]. VSM measurements indicate that the decrease in the GMR value is not due to temperature dependence of $F_{\mathrm{AF}}$ since in the as-deposited sample it does not change much within the investigated temperature range. Considerable and almost linear decrease in GMR effect saturation field $H_{\mathrm{s}}$ with increasing temperature (see the insert in Fig. 2) is probably due to temperature dependence of magnetization profile in direction normal to $\mathrm{Py} / \mathrm{Cu}$ interface which in turn can result in a decrease 
in an effective coupling energy [8]. It can also be argued that $H_{\mathbf{s}}$ changes because the effective thickness of non-magnetic spacer changes with temperature. In that case it would be a mixing at interfaces and consequent position dependence of a local Curie temperature that would cause outer monolayers of Py sublayers to loose magnetic moment at elevated temperature. In view of a strong dependence of GMR saturation field value in AF-coupled multilayers on spacer thickness [9] it should result in decrease (or increase) in $H_{\mathbf{s}}$. The first mechanism discussed seems however more probable since previous investigations indicate that the nominal $\mathrm{Cu}$ sublayer thickness for which local $H_{\mathrm{s}}$ maximum is observed changes with temperature much less than the thickness of magnetically inactive part of Py sublayers [10].

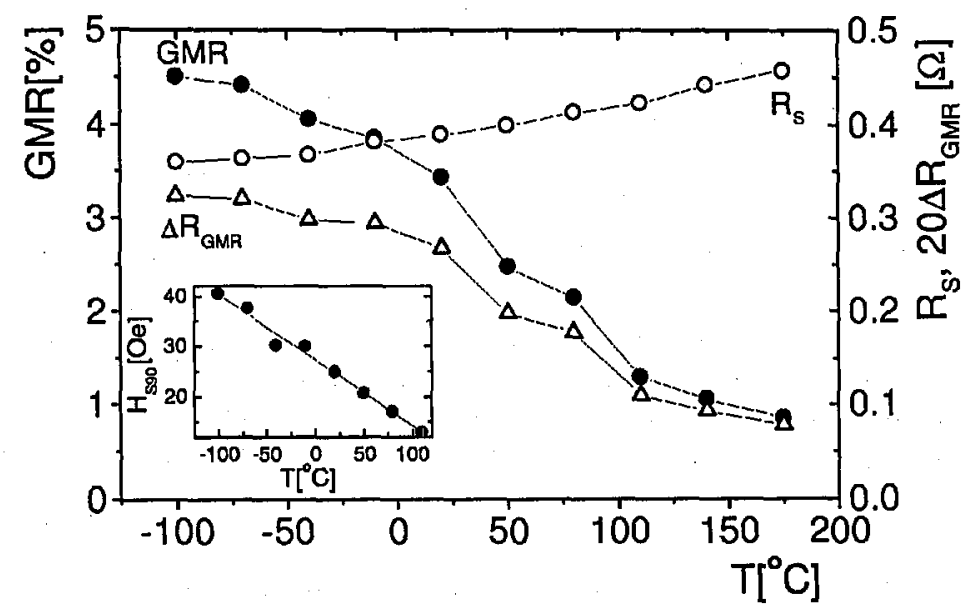

Fig. 2. Magnetoresistance ratio (solid circles), saturation resistance (open circles), resistance change $\Delta R_{\mathrm{GMR}}$ (triangles) and saturation field (insert) of $\mathrm{Si}(100) / \mathrm{Cu}(20 \mathrm{~nm}) /$ $\mathrm{Py}(2 \mathrm{~nm}) /(\mathrm{Cu}(2 \mathrm{~nm}) / \mathrm{Py}(2 \mathrm{~nm}))_{100}$ multilayer as a function of temperature. Magnetic field applied parallel to easy axis direction.

The series of 2 hour isothermal annealings resulted in a considerable increase in GMR ratio (Fig. 3). It is caused mainly by the increase in the $F_{\mathrm{AF}}$ ratio as indicated by approximately linear dependence of $\Delta R_{\mathrm{GMR}}$ on $F_{\mathrm{AF}}$ (see insert). Annealing led also to a $15 \%$ decrease in base resistance $R_{\mathrm{s}}$ and its influence on GMR ratio was comparable with that of increased $F_{\mathrm{AF}}$. Two basic mechanisms can be responsible for the increase in $F_{\mathrm{AF}}$ ratio. The first consists in $\mathrm{Cu}$ atoms diffusing into magnetic bridges that connect Py sublayers and couple them ferromagnetically. This could lead to the lowering of the local $T_{\mathrm{C}}$, annihilation of bridges and consequent increase in $F_{\mathrm{AF}}$. The second mechanism could be the diffusion driven break up of Py sublayers into discontinuous layers. In such case each bridge couples pancake-like Py granules and the area of its influence within the magnetic sublayer is limited. It is the so-called lateral decoupling. Complementary CEMS measurements provided no evidence for any significant changes in the microstructure of $\mathrm{Py} / \mathrm{Cu}$ interface in the course of thermal treatment. They excluded specifically the possibility of any significant volume changes in multilayer structure (e.g. Fe atoms moving from bulk to interface sites): They did not exclude 
however the possibility of some grain boundary diffusion which could lead to the annihilation of bridges. Hyperfine field values obtained were typical of permalloy $(24.5 \mathrm{~T})$.

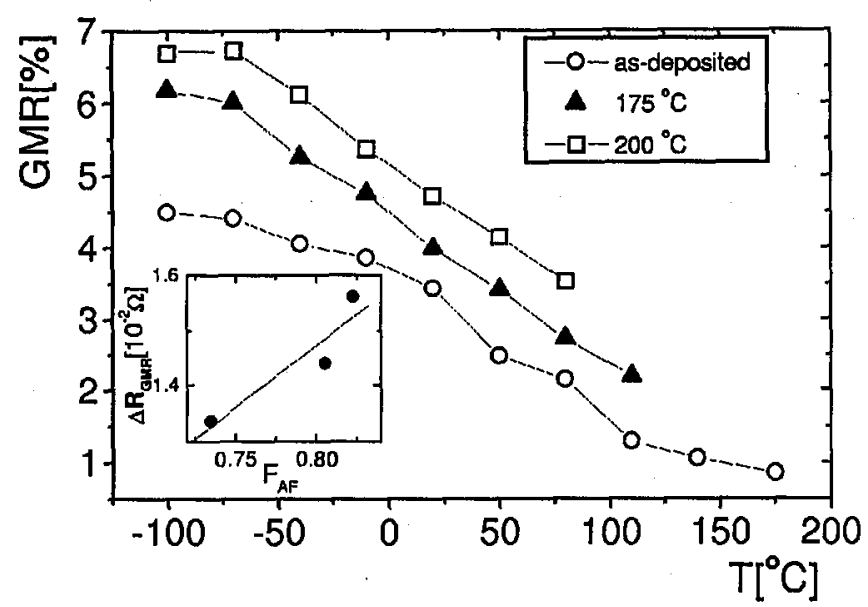

Fig. 3. Magnetoresistance ratio versus temperature for $\mathrm{Si}(100) / \mathrm{Cu}(20 \mathrm{~nm}) / \mathrm{Py}(2 \mathrm{~nm}) /$ $(\mathrm{Cu}(2 \mathrm{~nm}) / \mathrm{Py}(2 \mathrm{~nm}))_{100}$ multilayer after subsequent stages of thermal treatment (see legend). Insert: $\Delta R_{\mathrm{GMR}}\left(F_{\mathrm{AF}}\right)$ dependence for three stages of thermal treatment.

In conclusion, our results have shown a relatively good thermal stability of GMR effect in $\mathrm{Py} / \mathrm{Cu}$ multilayers from the second AF-region $\left(t_{\mathrm{Cu}}=2 \mathrm{~nm}\right)$. It was also shown that changes in the GMR ratio brought about by annealing are mainly caused by the consequent decrease in the effectiveness of the magnetic bridges connecting neighbouring permalloy layers.

\section{References}

[1] B: Dieny, J. Magn. Magn. Mater. 136, 335 (1994).

[2] M.A. Parker, K.R. Coffey, J.K. Howard, Ch.H. Tsang, R.E. Fontana, T.L. Hylton, IEEE Trans. Magn. 32, 142 (1996).

[3] M. Urbaniak, T. Luciński, F. Stobiecki, J. Magn. Magn. Mater. 190, 187 (1998).

[4] J.M. Daughton, J. Magn. Magn. Mater. 192, 334 (1999).

[5] T. Luciński, F. Stobiecki, Acta Phys. Pol. A 91, 273 (1997).

[6] J. Baszyński, F. Stobiecki, B. Szymański, K. Chrzumnicka, Phys. Status Solidi A 141, K23 (1994).

[7] J. Barnaś, Acta Phys. Pol. A 85, 165 (1994).

[8] R. Camley, R.L. Stamps, J. Phys., Condens. Matter. 5, 3727 (1993).

[9] T. Luciński, F. Stobiecki, D. Elefant, D. Eckert, G. Reiss, J. Magn. Soc. Japan 23, 126 (1999).

[10] J. Dubowik, F. Stobiecki, T. Luciński, Phys. Rev. B 57, 5955 (1998). 